\title{
Current Research Trends in Traditional Chinese Medicine Formula: A Bibliometric Review from 2000 to 2016
}

\author{
Yi-Bing Chen $\mathbb{D}^{1,2,3}$ Xiao-Fang Tong, ${ }^{1,2,3}$ Junge Ren, \\ Chun-Quan Yu ${ }^{D}$, 1 and Yuan-Lu Cui ${ }^{1,2,3}$ \\ ${ }^{1}$ Tianjin University of Traditional Chinese Medicine, Tianjin 301617, China \\ ${ }^{2}$ Research Center of Traditional Chinese Medicine, Tianjin University of Traditional Chinese Medicine, Tianjin 301617, China \\ ${ }^{3}$ Key Research Laboratory of Prescription Compatibility among Components, Tianjin University of Traditional Chinese Medicine, \\ Tianjin 301617, China \\ ${ }^{4}$ Tianjin University Library, Tianjin 300072, China
}

Correspondence should be addressed to Chun-Quan Yu; ycq-4@163.com and Yuan-Lu Cui; cuiyl@tju.edu.cn

Received 3 November 2018; Accepted 13 February 2019; Published 3 March 2019

Academic Editor: Armando Zarrelli

Copyright (C) 2019 Yi-Bing Chen et al. This is an open access article distributed under the Creative Commons Attribution License, which permits unrestricted use, distribution, and reproduction in any medium, provided the original work is properly cited.

\begin{abstract}
Background. Traditional Chinese Medicine Formula (TCMF) study has been recognized widely by medical scientists around the world. However, few researchers have analyzed and summarized the rapid growth of academic articles of TCMF published in English. The primary aim of this work was to assess the outcome of these research outputs in the TCMF field from 2000 to 2016 and to evaluate the situation and tendency. Methods. Research datasets were acquired from the Web of Science database, which includes all academic articles published from 2000 to 2016; articles were tracked by the keywords "Traditional Chinese Medicine", "Traditional Chinese Medicine Formula", and "Chinese herb formula". Moreover, visualization software CiteSpace V was used to analyze and generate visualization knowledge maps. Results. In total, 26,917 articles appeared in the Web of Science database, and only 2,621 publications met requirement based on reading the abstract or full text. The annual publications total, list of journals, research interests, list of medicine names, disease types, and the top 20 cited articles were given in this research paper. In addition, we compared the research of Japan and Korea TCMF, in the appendix. Conclusion. This review demonstrates that increasingly more researchers have interest in the TCMF and TCMF has great significant advantages over other areas of focus. However, these publications were published rarely in top academic journals and most best-quality papers have bias toward medical analysis rather than pharmacology. To make a breakthrough in TCMF field, further investigation is required to place emphasis on the deepening study of the mechanism of related TCMF.
\end{abstract}

\section{Background}

Advances in modern science and technology have contributed greatly to the development of the pharmaceutical industry and clinical medicine. However, East Asian countries are still generally using Traditional Chinese Medicine due to the impact on culture and history. The WHO has asserted that the Traditional Medicine is one of the primary sources of healthcare [1]. Furthermore, because of Prof. Youyou Tu's extraordinary achievement in using artemisinin treatment, she has been horned with the Physiology or Medicine Award from the Nobel Prize Organization in 2015. Hence, Traditional Chinese Medicine has attracted increasingly more attention in the global medical community
[2]. In general, the Traditional Chinese Medicine Formula (TCMF) has two or more kinds of herbs as components, is designed for relatively certain symptoms, and is an important measure of the Chinese medicine treatment of diseases [3]. Knowledge regarding TCMF was not accepted in the Western Society in earlier years [4]; hence it was very hard to find the articles in peer-reviewed journals. Fortunately, the Chinese government constantly has supported the TCMF field, for example, the approval of the State Council, National Science \& Technology Major Project of China "Key New Drug Creation and Manufacturing Program" launched in 2008. Thus, at a turning point, the year of 2010, the status of the Traditional Chinese Medicine has improved greatly, and the TCMF eventually has gained its deserved reputation. 
Bibliometrics is a statistical analysis of written publications [5], and it is also a useful tool and method to evaluate or summarize research results in the particular field [6]. The vital indicator of estimating the quality of one article is citation rate [7]; citation analysis is necessary to provide a multidimensional summary for articles in a specific field. In addition, the TCMF research field is rich in intrinsic connection and external divergence because of its own characteristics. CiteSpace, an appropriate piece of software and a useful tool that focuses on visualizing and analyzing trends and patterns in scientific literature, was applied to conduct bibliometric analysis [8]. The results of the analysis are important for the future research of TCMF. Therefore, this paper considers bibliometrics from the perspective of article and citation analysis, which will help guide researchers or research funding agencies toward areas where there is lack of focus on research activity and provide the reader with insight and valuable information.

\section{Methods}

2.1. Search Strategy. Papers published from 2000 to 2016 were tracked by the keywords "Traditional Chinese Medicine", "Traditional Chinese Medicine Formula", and "Chinese Herb Formula" for inclusion in analysis and summarization, based on their presence in the Web of Science database. Web of Science is an online subscription-based scientific citation indexing service originally produced by the Institute for Scientific Information. It is the largest comprehensive academic information resource with the largest number of disciplines. It contains more than 12,000 core academic journals with the most influential research fields in natural sciences, engineering technology, and biomedicine [9].

During the article-collecting process, we encountered some difficulties such as "inaccurate screening result"-some formulae abbreviations or herb-pairs appear in the articles but without a detailed-explaining-and "undesired document type"-original and review articles are the only document types in this study, and meeting, editorial, and other document types are nontarget samples. Hence, these "biased articles" were not included in our finalized dataset. Therefore, the statistical analysis would be more trustworthy and accurate. The citations were gathered within one day on December 31, 2017, to avoid the possibility of unfairness due to the daily database update. The papers in this TCMF analysis were published in English in many international journals, all the authors are from China (including Mainland, Hong Kong, Macao, Taiwan), and the first author's region was determined by his/her organization regardless of citizenship and residence status.

2.2. Data Analysis. The papers search from the Web of Science were transferred to EndNote X7 (Thomson Reuters, San Francisco, CA) for classification and statistical analysis. Basic information such as year of publications, journals, region, and citations was recorded directly for analysis. The following information, research interest, medicine name, and disease type, was reviewed by reading the abstract or full text for statistics. Finally, the result sorting process was generated by Microsoft Office Excel ${ }^{\circledR}$ 2007. The categorical data are presented as integer and percentages. CiteSpace $\mathrm{V}$ was used to visualize and verify the high-frequency keywords, categories, and authors in a graphical way [10]. The parameters of CiteSpace V were as follows: time slicing (2000-2016), years per slice (1), term source (all selection), node type (choose one at a time), selection criteria (top 50), and pruning (pathfinder).

With the purpose of conducting deeper research and evaluating the international recognition of TCMF, we also screened TCMF-related articles from Japanese and South Korean publications by using the same methodology and then alphabetized the TCMF in a descending order (Z-A) according to Chinese Pinyin, Japanese Romaji, and South Korean Romaji. Please refer to the Supplementary Materials for details.

\section{Results}

A total of 26,917 TCMF-related articles were published in the period from 2000 to 2016. Refining conditions and reading the abstract or the full text aim to reduce statistical bias. As a result, a total of 2,621 articles were used from the search strategy reported above. Among these articles, 2,451 were original articles and 170 were review articles. Figure 1 showed the search methodology and the corresponding results. Moreover, 2,233 articles were published in Mainland China, 273 were published in Taiwan, 107 were published in Hong Kong, and 8 were published in Macao.

To begin with the statistical analysis, 1,345 articles used animals as the research objects, 406 articles used cells as the research objects, and 383 used patients as the research objects. Certainly, many pharmacological studies are not only on animals but also on cells.

3.1. Tendency of Article Publication. The number of annual publications in 2000 to 2016 was given in Table 1. It indicated that almost $63 \%$ of articles were published during the recent four years (2013-2016). Regarding such limitations, considering that there is a language barrier and less knowledge about the Traditional Chinese Medicine, TCMF-related articles were rarely published in international journals from 2000 to 2004. Due to the Chinese government support and the research's efforts and exploration, notably, the annual publication amount has gradually increased. Table 2 showed that there was an obvious two-sided preference between the journals and TCMF researchers; among all these TCMF publications, approximately $25 \%$ were from the Journal of Ethnopharmacology and Evidence-Based Complementary and Alternative Medicine. The articles published in these top 20 journals account for $56.1 \%$ of the total.

3.2. Research Content. Moreover, Table 3 showed the research interests of research production and Table 4 listed the top 20 popular disease types. Pharmacology was the trendiest research interest in the TCMF field of study; among these 2,621 papers, approximately $1,515(57.80 \%)$ papers were pharmacology related, followed by pharmaceutical analysis (691, $26.36 \%)$ and clinical research $(381,14.54 \%)$. More than 300 


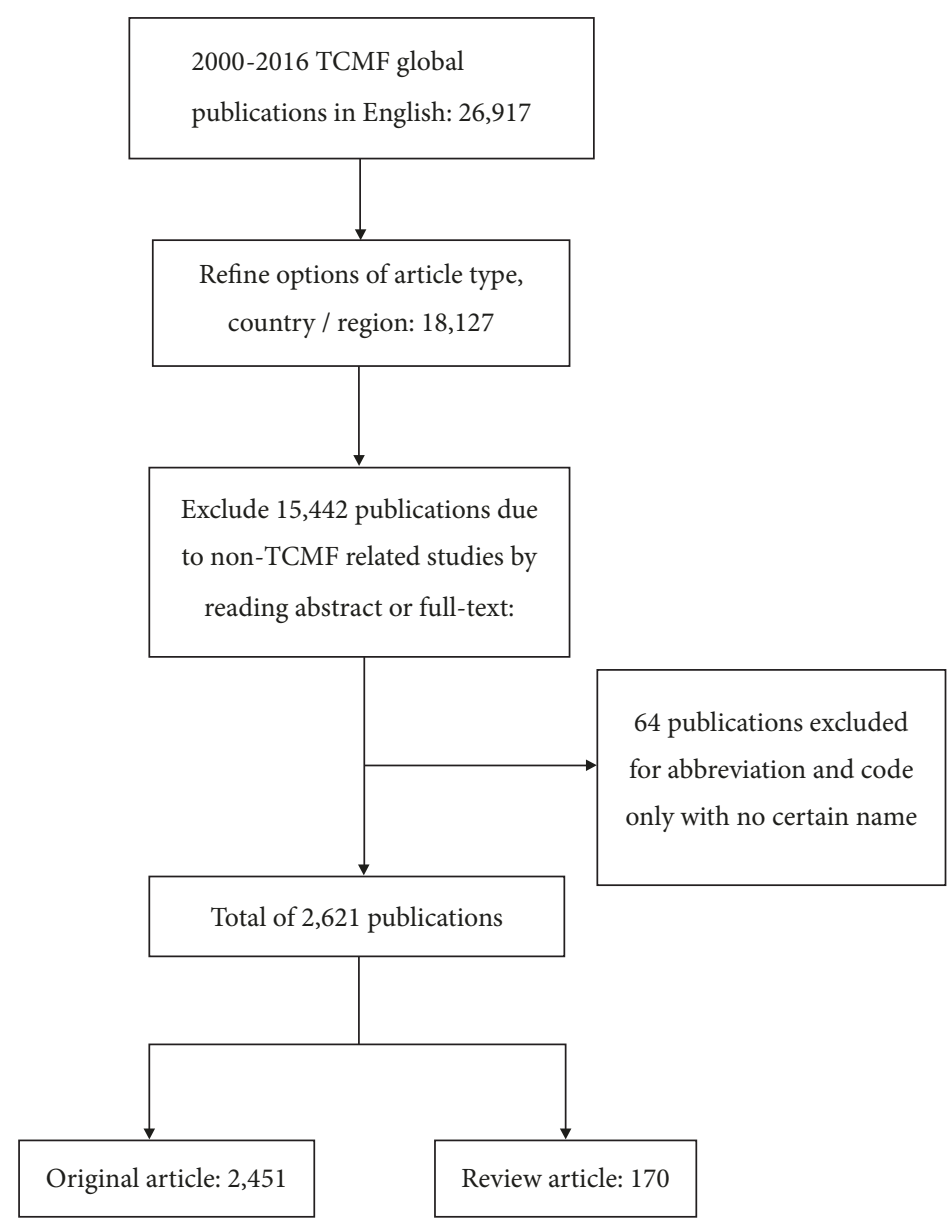

FIGURE 1: Flow chart of publications search and study selection.

TABLE 1: The number of annual publications of Traditional Chinese Medicine Formula related articles from 2000 to 2016.

\begin{tabular}{lcc}
\hline Year & Number & Percentage( /2621) \\
\hline 2016 & 478 & $18.23 \%$ \\
2015 & 450 & $17.17 \%$ \\
2014 & 380 & $14.50 \%$ \\
2013 & 344 & $13.12 \%$ \\
2012 & 212 & $8.09 \%$ \\
2011 & 180 & $6.87 \%$ \\
2010 & 128 & $4.88 \%$ \\
2009 & 104 & $3.97 \%$ \\
2008 & 92 & $3.51 \%$ \\
2007 & 67 & $2.56 \%$ \\
2006 & 63 & $2.40 \%$ \\
2005 & 42 & $1.60 \%$ \\
2004 & 24 & $0.92 \%$ \\
2003 & 23 & $0.88 \%$ \\
2002 & 12 & $0.46 \%$ \\
2001 & 9 & $0.34 \%$ \\
2000 & 13 & $0.50 \%$ \\
\hline
\end{tabular}

disease types were mentioned in the datasets, and Table 4 showed that cardiovascular disease and cancer were mostly discussed. The subcategories of cardiovascular disease and cancer types are also listed in detail in Table 5.

Table 6 is a list of top 20 TCMF names, traditional herb formulae, and modern proprietary Chinese medicines, including more than 800 summarized formulae. Specifically, Huang Lian Jie Du had been studied in 62 publications, followed by Bu Yang Huan Wu Tang (49) and Shen Fu injection (44). Table 7 shows the top 20 highly cited TCMFrelated articles.

3.3. CiteSpace Visualization Analysis. CiteSpace V was used to generate a keyword map, category map, and coauthor map. The top 25 keywords and categories with the strongest citation burst were also drawn.

Combining Figures 2 and 3, an analysis in terms of keyword counts, "HPLC" and "quality control", indicated that the researchers may have greater research interests in drug chemical analysis and drug composition identification during 2004-2011; flavonoids, such as baicalin and berberine, and glycosides, such as paeoniflorin, were prominent from 2004 to 2011. These Traditional Chinese Medicine monomers above as natural medicine were highly recognized; their excellent antioxidation effects are also the main mechanism of many pharmacological studies [11-14]. 
TABLE 2: List of top 20 journals in which articles were published and the newest impact factor (IF)* ${ }^{*}$

\begin{tabular}{|c|c|c|c|}
\hline Rating & Journal & Number & IF \\
\hline 1 & Journal of Ethnopharmacology & 379 & 3.115 \\
\hline 2 & $\begin{array}{c}\text { Evidence-Based Complementary and } \\
\text { Alternative Medicine }\end{array}$ & 247 & 2.064 \\
\hline 3 & $\begin{array}{c}\text { Journal of Pharmaceutical and } \\
\text { Biomedical Analysis }\end{array}$ & 99 & 2.831 \\
\hline 4 & $\begin{array}{l}\text { BMC Complementary and Alternative } \\
\text { Medicine }\end{array}$ & 78 & 2.109 \\
\hline 5 & PLOS One & 66 & 2.766 \\
\hline 6 & Journal of Separation Science & 56 & 2.415 \\
\hline 7 & Chinese Journal of Integrative Medicine & 55 & 1.346 \\
\hline 8 & Biomedical Chromatography & 53 & 1.688 \\
\hline 9 & American Journal of Chinese Medicine & 50 & 3.120 \\
\hline 10 & Journal of Traditional Chinese Medicine & 50 & 0.857 \\
\hline 11 & $\begin{array}{c}\text { Journal of Chromatography B-Analytical } \\
\text { Technologies in the Biomedical and Life } \\
\text { Sciences }\end{array}$ & 45 & 2.441 \\
\hline 12 & Neural Regeneration Research & 42 & 2.234 \\
\hline 13 & Scientific Reports & 40 & 4.122 \\
\hline 14 & Experimental and Therapeutic Medicine & 35 & 1.410 \\
\hline 15 & Molecules & 37 & 3.098 \\
\hline 16 & Chinese Medical Journal & 32 & 1.596 \\
\hline 17 & Molecular Medicine Reports & 30 & 1.922 \\
\hline 18 & Trials & 27 & 2.067 \\
\hline 19 & Analytical Methods & 26 & 2.073 \\
\hline 20 & Chromatographia & 25 & 1.401 \\
\hline
\end{tabular}

* The impact factor was reported according to Institute for Scientific Information (ISI) journal citation reports (JCR) 2018.

TABLE 3: Research interests of articles by reading abstracts or fulltexts.

\begin{tabular}{lc}
\hline Research interests & Number (\%)* \\
\hline Pharmacology & $1515(57.80 \%)$ \\
Pharmaceutical analysis & $691(26.36 \%)$ \\
Clinical research & $381(14.54 \%)$ \\
Mathematical Analysis and System Biology & $64(2.44)$ \\
Toxicology & $32(1.22 \%)$ \\
Pharmaceutical preparations & $27(1.03 \%)$ \\
Pathology & $4(0.02 \%)$ \\
\hline
\end{tabular}

* Total percentage exceeds $100 \%$ due to multidiscipline interaction.

Category could help researchers understand the focus of TCMF research, combining Figures 4 and 5, immunology, pharmacology, and drug analysis were featured in the early stage, but research on the cardiovascular system was in-depth from 2009 to 2014, and the focus of research from 2014 to 2016 shifted to biophysics, science and technology, and other multidisciplinary sciences.

Figure 6 shows the coauthor map; this analysis result revealed the cooperative relationship between the authors. Coauthor analysis can be used as an important tool in the evaluation of bibliometrics to identify research groups in "unknown" universities or organizations [15].

\section{Discussion}

This report analyzed TCMF-related articles published in international journals from 2000 to 2016 . To our knowledge, this is the first report that analyzed the quality and quantity of TCMF-related studies in China. More than 26,000 articles were obtained when using "Traditional Chinese Medicine", "Traditional Chinese Medicine Formula", and "Chinese herb formula" as the keywords in a search of the Web of Science database. A total of 2,621 articles were screened out by reading the abstract or full text. Each database has its own advantages and characteristics. A possible explanation for choosing the Web of Science as the only database is that it offered several advantages: First of all, Web of Science, with high recognition and article quality for many years, offers a powerful analysis of data from various aspects and the selected articles. Second, Web of Science has a function of EndNote online. During the items collecting process, the documents that meet the requirements could be added to the EndNote online at any time and there was logo displayed on the web pages. This unique feature could easily transfer the articles to the EndNote software in order to conduct the future analysis. Third, every database has its own standard on calculating the citation rate of article and evaluating the importance and influence of specific publications. Hence, choosing Web of Science could guarantee a powerful uniform citation rate and impact of journals. 
TABLE 4: List of the top 20 types of disease appearing in articles

\begin{tabular}{lcc}
\hline Rating & Disease types & Number \\
\hline 1 & Cardiovascular disease & 335 \\
2 & Cancer & 156 \\
3 & Diabetes & 105 \\
4 & Depression & 108 \\
5 & Alzheimer's disease & 48 \\
6 & Asthma \\
7 & Liver fibrosis (hepatic fibrosis) \\
8 & Rheumatoid arthritis \\
9 & Osteoporosis \\
10 & Liver injury \\
11 & Dementia \\
12 & Diabetic nephropathy \\
13 & Chronic obstructive pulmonary disease \\
14 & Osteoarthritis \\
15 & Insomnia \\
16 & Ulcerative colitis \\
17 & Fatigue \\
18 & Parkinson's disease \\
19 & Headache \\
20 & Dysmenorrhoea & 33 \\
\hline
\end{tabular}

TABLE 5: The top types of cardiovascular diseases and cancers.

\begin{tabular}{lccc}
\hline Cardiovascular disease & Number & Cancer & Number \\
\hline Ischemic stroke & 37 & Anti-tumor & 33 \\
Hypertension & 33 & Breast cancer & 25 \\
Myocardial infarction & 29 & Colorectal cancer & 21 \\
Heart failure & 29 & Hepatocellular carcinoma & 14 \\
Stroke & 29 & Lung cancer & 13 \\
Atherosclerosis & 24 & Gastric cancer & 12 \\
Chronic heart failure & 14 & Liver cancer & 10 \\
Sepsis & 14 & Bone cancer \\
Coronary heart disease & 13 & Leukemia \\
Cardiac arrest & 12 & Colon cancer \\
Angina & 10 & & 4 \\
\hline
\end{tabular}

Chinese medicine culture has existed in China for thousands of years, since the era of ancient China; countless research studies have been written by Chinese medicine scholars. However, due to the limited ability of research and the language barrier, TCMF publications were not favored in the international journals. Fortunately, the amount of TCMF publications has increased remarkably over time, especially after 2010. With support from Chinese government and the development of science and technology, we do observe increasingly more scientific research outputs from the medicine community. A significant contribution to the TCMF development occurred when Prof. Youyou Tu won the Physiology or Medicine Noble Prize [2], which encourages Traditional Chinese Medicine professionals to explore more about the TCMF research contents. Thus, a breakthrough in the TCMF field of research is the key to a brighter future for the medicine community.

According to the preparatory work, TCMF-related articles have been published in more than 300 journals. Most of the TCFM articles were published in the Journal of Ethnopharmacology and Evidence-Based Complementary and Alternative Medicine, which matches the result from Sa'ed $\mathrm{H}$. Zyoud's bibliometric analysis of the Integrative and Complementary Medicine field [16]. This means that the Journal of Ethnopharmacology and Evidence-Based Complementary and Alternative Medicine are more likely to accept Traditional Medicine articles, when compared with the other publications.

Furthermore, pharmacology study has been recorded as the most popular research filed; 1,515 out of 2,621 articles 
TABle 6: The top 20 popular Traditional Chinese Medicine Formulas involved in articles.

\begin{tabular}{lcc}
\hline Rating & Traditional Chinese Medicine Formula & Number \\
\hline 1 & Huang Lian Jie Du tang & 62 \\
2 & Bu Yang Huan Wu tang & 49 \\
3 & Shen Fu injection & 44 \\
4 & Sheng Mai san & 44 \\
5 & Liu Wei Di Huang wan & 42 \\
6 & Si Wu tang & 41 \\
7 & Da Cheng Qi tang \\
8 & Dang Gui Bu Xue tang \\
9 & Tong Xin Luo capsule \\
10 & Si Ni san \\
11 & Xue Fu Zhu Yu tang \\
12 & Kai Xin san \\
13 & Xiao Chai Hu tang \\
14 & Xiao Yao san & 37 \\
15 & Xie Xin tang \\
16 & Pien Tze Huang \\
17 & Ge Gen Qin Lian tang \\
18 & Jia Wei Xiao Yao San \\
19 & Bu Zhong Yi Qi tang \\
20 & Dan Hong injection & 29 \\
\hline
\end{tabular}

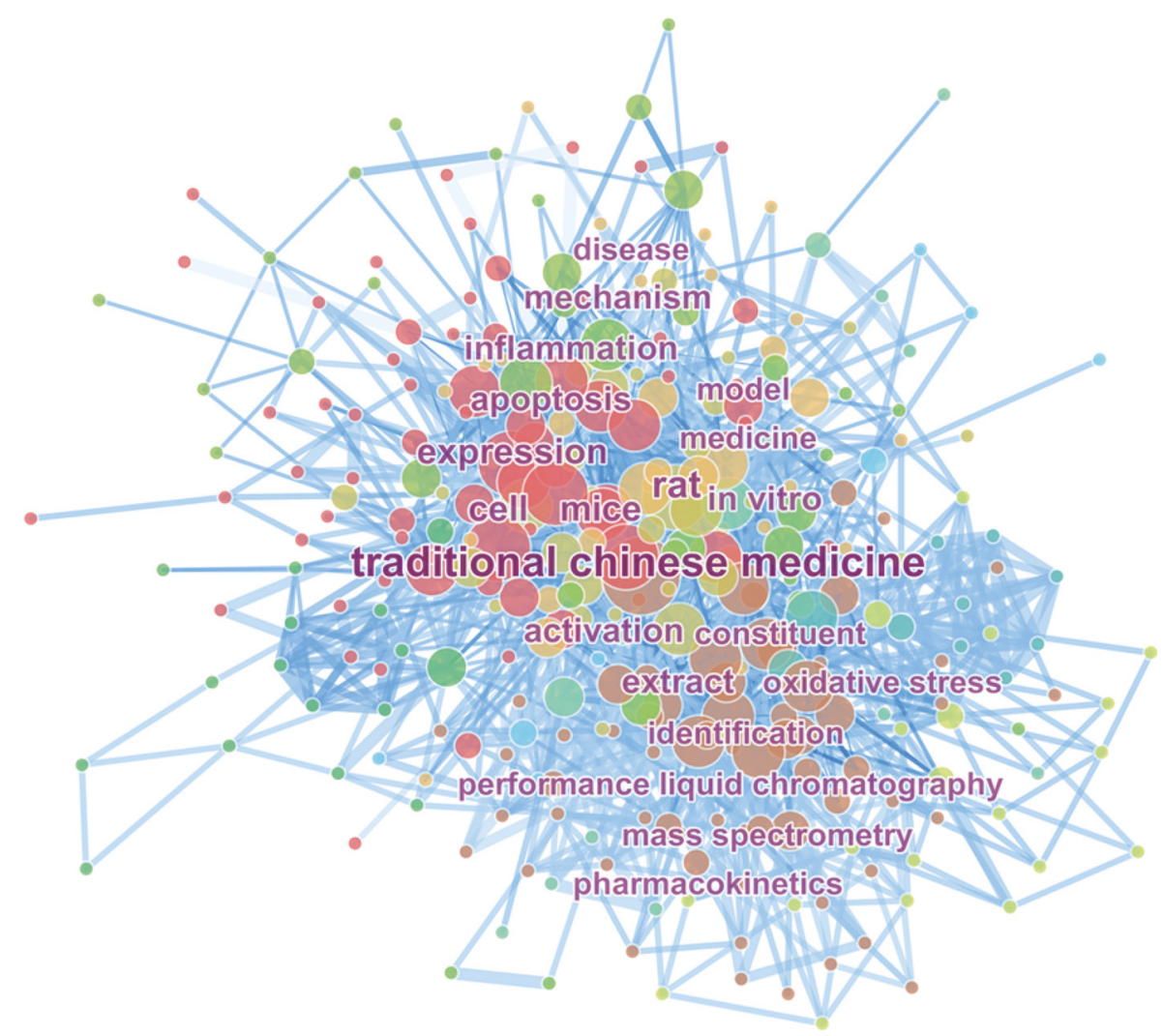

FIGURE 2: Keyword map related to TCMF research from 2000 to 2016. A total of 308 nodes and 1521 connection lines were obtained. The size and position of nodes represent research frequency and the level of core of the keyword, respectively. The darkness of blue line represents the different years. 


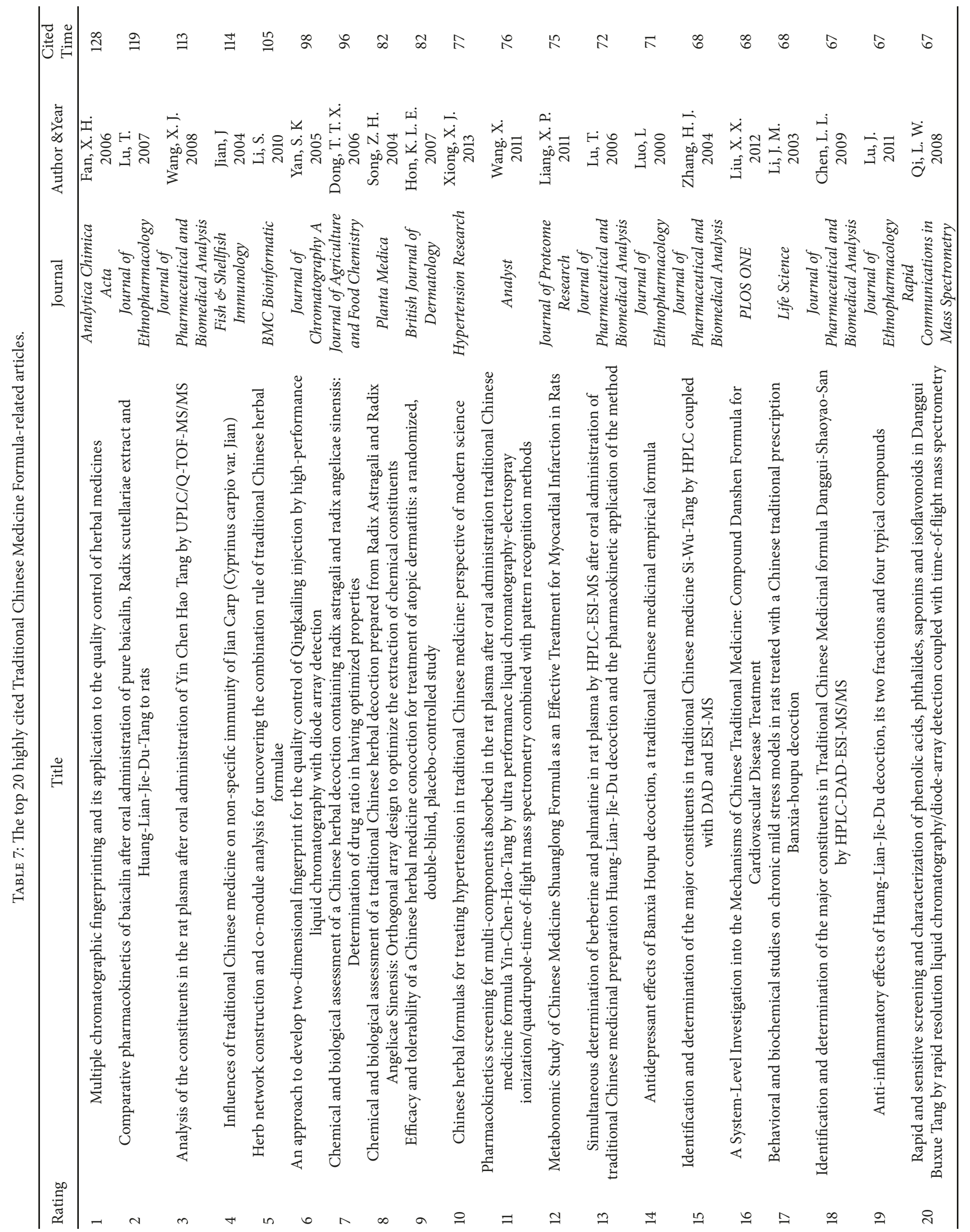




\begin{tabular}{|c|c|c|c|c|c|}
\hline Keywords & Year & Strength & Begin & End & $2000-2016$ \\
\hline hplc & 2000 & 11.8395 & 2004 & 2011 & \\
\hline gene expression & 2000 & 8.2585 & 2006 & 2011 & \\
\hline column liquid chromatography & 2000 & 8.1642 & 2007 & 2009 & \\
\hline performance liquid chromatography & 2000 & 7.9162 & 2006 & 2009 & \\
\hline berberine & 2000 & 7.822 & 2006 & 2011 & \\
\hline paeoniflorin & 2000 & 7.3268 & 2006 & 2013 & \\
\hline differentiation & 2000 & 6.9475 & 2006 & 2010 & \\
\hline angelica sinensis & 2000 & 6.8701 & 2004 & 2009 & \\
\hline quality control & 2000 & 6.7991 & 2004 & 2011 & \\
\hline traditional Chinese medicine & 2000 & 6.4534 & 2005 & 2013 & \\
\hline Qingkailing injection & 2000 & 5.5349 & 2005 & 2007 & \\
\hline cytokine & 2000 & 5.0529 & 2007 & 2011 & \\
\hline buyang huanwu decoction & 2000 & 4.337 & 2006 & 2011 & \\
\hline antioxidant & 2000 & 4.2225 & 2004 & 2009 & \\
\hline flavonoid & 2000 & 4.134 & 2005 & 2009 & \\
\hline saponin & 2000 & 3.9024 & 2006 & 2011 & \\
\hline fingerprint & 2000 & 3.885 & 2006 & 2009 & \\
\hline efficacy & 2000 & 3.885 & 2006 & 2009 & \\
\hline root & 2000 & 3.8134 & 2006 & 2007 & \\
\hline inhibitor & 2000 & 3.2994 & 2005 & 2011 & \\
\hline separation & 2000 & 3.1771 & 2006 & 2007 & \\
\hline baicalin & 2000 & 3.1653 & 2004 & 2009 & \\
\hline estrogen & 2000 & 3.0666 & 2008 & 2009 & \\
\hline diode array detection & 2000 & 3.0666 & 2008 & 2009 & \\
\hline stress & 2000 & 3.0084 & 2003 & 2007 & \\
\hline
\end{tabular}

Figure 3: Top 25 keywords with the strongest citation burst. The red bars and blue bars demonstrate that some keywords are cited frequently and infrequently in a certain period.

discussed pharmacology. Pharmaceutical analysis is the second favorable topic with 691 out of 2.621 articles; 381 out of 2,621 articles had a clinical research focus. In pharmaceutical analysis study, the top three trendy topics are metabolomics, pharmacokinetics, and quality control. As reported in our database, there are 173, 167 and 82 articles conducted on these three areas, respectively. Fifty-one pharmacological analysis articles were included for the clinical study. The secondary major research concentration on clinical study was metaanalysis; 54 related articles were included. We have listed all the TCMF research topics that were being discussed widely. We hope this information will be helpful for future study.

Many of diseases could be cured through the TCMF treatment. For instance, approximately, 300 diseases that use TCMF methodology for treatments were mentioned in screening publications. Furthermore, studies on cardiovascular disease and cancer are mostly being analyzed, followed by diabetes and depression. A possible reason for this would be that cardiovascular disease and cancer are the most fatal diseases [17-19]. With the increasing pressure of modern life and the irregular lifestyles, diabetes and depression have become increasingly threatening to human health [20-23].

Countless Traditional Chinese Medicine and Chinese patent medicines are in the historical records; meanwhile, these medicines are also being used widely in the market. As noted, more than 800 types of Traditional Chinese Medicine were included in the database. The reason that Huang Lian Jie Du Tang was mentioned broadly is that the major three components of this medicine, Coptis chinensis Franch, Scutellaria baicalensis Georgi, and Phellodendron chinense Schneid, all contain flavonoid and alkaloid. Flavonoid and alkaloid work well for an anti-inflammatory effect $[24,25]$. Huang Lian Jie Du Tang is an effective treatment for inflammation [26], and inflammatory actions lead to many disorders [27-29]. Tonic 


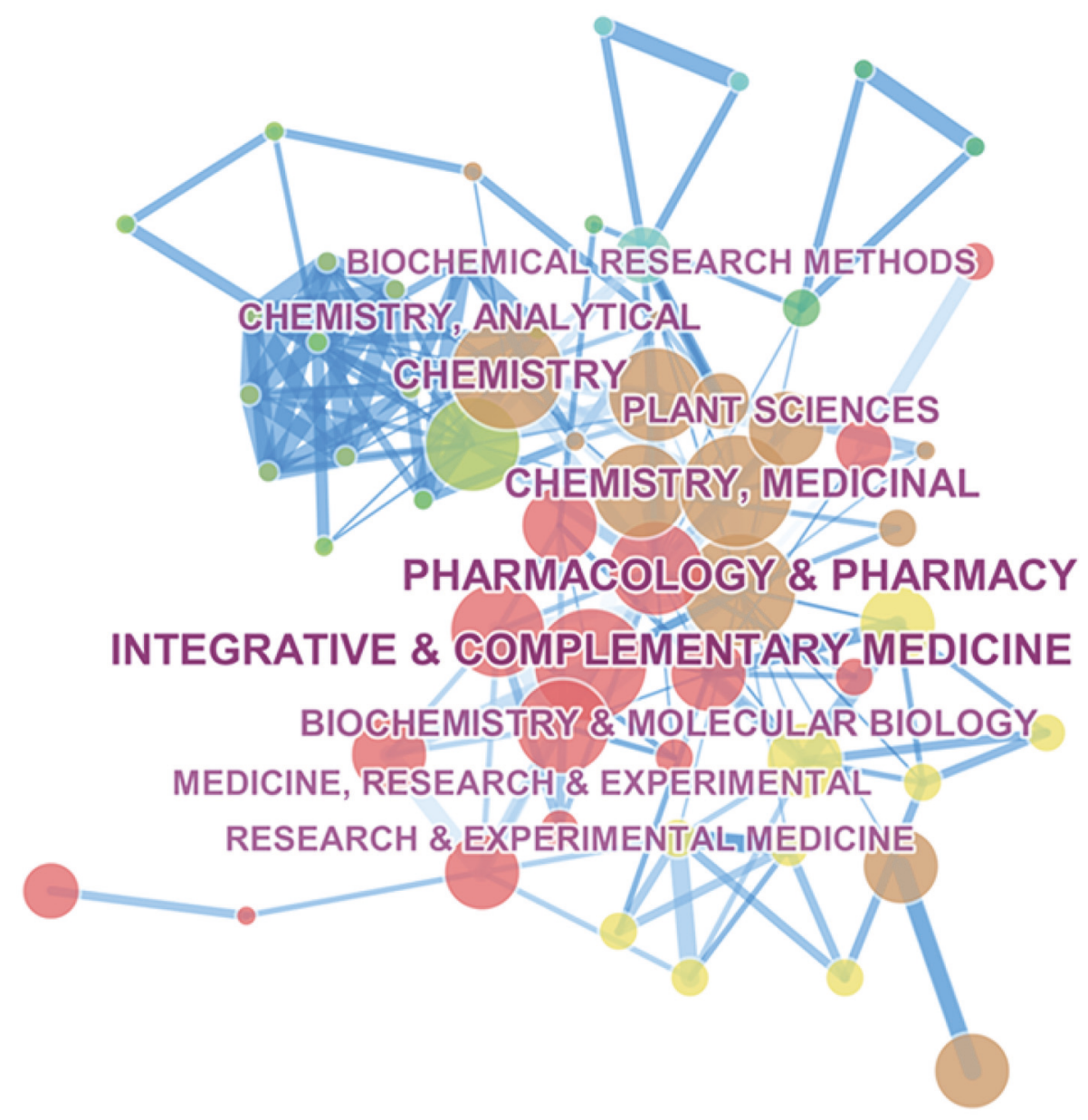

Figure 4: Category map related to TCMF research from 2000 to 2016. A total of 66 nodes and 194 connection lines were obtained. The size and position of nodes represent research frequency and the level of core of the category, respectively. The darkness of blue line represents the different years.

formulae, such as Sheng Mai san, Liu Wei Di Huang Wan, and Si Wu Tang, and blood-regulating formulae, such as Bu Yang Huan Wu tang and Xue Fu Zhu Yu tang, were listed in Table 6 as the top 20 most popular TCMF, and most of these formulae could be used in cardiovascular and cancer treatments [30-34]. Moreover, other formulae such as Kai Xin San and Xiao Yao San are commonly used for mental illness treatment $[35,36]$, and Dang Gui Bu Xue Tang and Huang Lian Jie Du Tang could also be adopted for diabetes treatment $[37,38]$. Thus, from these findings we can establish a clear mutual relationship between the tendency of TCMF studies and common diseases.

From Table 7, we can see that pharmaceutical analysis was the most frequently cited term in TCMF articles; this article previously mentioned that pharmacology is the most favorable research topic in TCMF study. Indeed, some respected journals such as Analytica Chimica Acta, Chromatography $A$, and Analyst are subject to the Chemistry section in the Science Citation Index. This evidence illuminates a strong argument that pharmaceutical analysis subject can be highly transferable and widely adopted in other fields. At the same time, some formulae were discussed frequently by TCMF scholars such as Huang Lian Jie Du Tang, Si Wu Tang, Dang Gui Bu Xue Tang, and Liu Wei Di Huang Wan; these are all included in the highly cited papers, as we previously mentioned. It should be mentioned that the Yin Chen Hao Tang was not included in Table 6, but there were still two highly cited articles that gave a detailed analysis of the formula. There are five pharmacological articles listed in Table 7; among them, one article is using Compound Dan Shen Formula in cardiovascular disease, and two articles are the application of Ban Xia Hou Po Tang in depression treatments. These were also diseases garnering a high level of attention. The above analysis demonstrated that research content and research interest, compound name, and disease type of TCMF were inseparable, which can provide a comprehensive understanding of the current development of TCMF.

Knowledge domain visualization, and visualization of scientific literature can strengthen the scientific and objective nature of bibliometrics and meet more practical needs, such as helping researchers analyze potential knowledge contained in scientific analysis $[39,40]$. In this paper, the bibliometric research of TCMF placed a particular emphasis on the statistics of research content and quantity. The subjective 
Top 25 Subject Categories with the Strongest Citation Bursts

\begin{tabular}{|c|c|c|c|c|c|}
\hline Subject Categories & Year & Strength & Begin & End & $2000-2016$ \\
\hline IMMUNOLOGY & 2000 & 8.5516 & 2000 & 2006 & \\
\hline PHARMACOLOGY \& PHARMACY & 2000 & 7.6125 & 2000 & 2004 & \\
\hline ALLERGY & 2000 & 4.1809 & 2000 & 2006 & \\
\hline TOXICOLOGY & 2000 & 2.5308 & 2000 & 2003 & \\
\hline FOOD SCIENCE \& TECHNOLOGY & 2000 & 1.6091 & 2000 & 2005 & \\
\hline CHEMISTRY, MULTIDISCIPLINARY & 2000 & 1.8437 & 2002 & 2004 & \\
\hline MEDICINE, GENERAL \& INTERNAL & 2000 & 9.1122 & 2003 & 2007 & \\
\hline GENERAL \& INTERNAL MEDICINE & 2000 & 8.9386 & 2003 & 2007 & \\
\hline GASTROENTEROLOGY \& HEPATOLOGY & 2000 & 8.1717 & 2004 & 2011 & \\
\hline CHEMISTRY, ANALYTICAL & 2000 & 4.6517 & 2006 & 2007 & \\
\hline CHEMISTRY & 2000 & 3.1653 & 2006 & 2007 & \\
\hline BIOCHEMICAL RESEARCH METHODS & 2000 & 8.8431 & 2007 & 2009 & \\
\hline NEUROSCIENCES \& NEUROLOGY & 2000 & 4.1248 & 2008 & 2009 & \\
\hline NEUROSCIENCES & 2000 & 3.5996 & 2008 & 2009 & \\
\hline PERIPHERAL VASCULAR DISEASE & 2000 & 2.072 & 2008 & 2009 & \\
\hline CARDIOVASCULAR SYSTEM \& CARDIOLOGY & 2000 & 2.4487 & 2009 & 2014 & \\
\hline AGRICULTURE & 2000 & 2.1965 & 2009 & 2010 & \\
\hline CHEMISTRY, MEDICINAL & 2000 & 2.9139 & 2010 & 2011 & \\
\hline CARDIAC \& CARDIOVASCULAR SYSTEMS & 2000 & 2.2123 & 2010 & 2014 & \\
\hline VETERINARY SCIENCES & 2000 & 1.5346 & 2010 & 2012 & \\
\hline MULTIDISCIPLINARY SCIENCES & 2000 & 7.6532 & 2014 & 2016 & \\
\hline SCIENCE \& TECHNOLOGY - OTHER TOPICS & 2000 & 7.1502 & 2014 & 2016 & \\
\hline BIOPHYSICS & 2000 & 2.5563 & 2014 & 2016 & \\
\hline BIOLOGY & 2000 & 1.825 & 2014 & 2016 & \\
\hline PSYCHIATRY & 2000 & 1.8232 & 2014 & 2016 & \\
\hline
\end{tabular}

Figure 5: Top 25 categories with the strongest citation burst. The red bars and blue bars mean some keywords are cited frequently and infrequently in a certain period.

analysis in the work could be supplemented with the CiteSpace $\mathrm{V}$ software, and the literature bibliometric research of TCMF could offer more in-depth and comprehensive information.

There are many articles on the research of the TCMF, whether in Asian countries or Western countries, but our work mainly analyzes the published trend of the articles on TCMF in China and concerned part of other Asian countries in recent years, which does not involve Western countries, because China is in the majority in leading this research so far. Moreover, most of the research on TCMF has been published in Chinese, and Chinese papers were not included in this study. However, according to the scientific research management orientation in recent years in China, excellent research results have been published in English journals. Therefore, this study has included the most important research which is representative of TCMF. Nevertheless, we still hope to see that more and more domestic and foreign scholars can pay attention to the study of TCMF.

From 2013 to 2016, TCMF study was being improved greatly by the Traditional Chinese Medicine researchers. For instance, the TCMF articles published in this period account for $63 \%$ of the total article amount. This scenario indicates that increasingly more scholars have interest in TCMF study. Further investigation is required to focus on the treatment of certain diseases such as cardiovascular disease and cancers. A deeper understanding and knowledge of each Traditional Chinese Medicine's component is essential because of the complexity and particularity of TCMF. Moreover, cooperating and communicating with scholars from other countries would boost the development of TCMF study. 


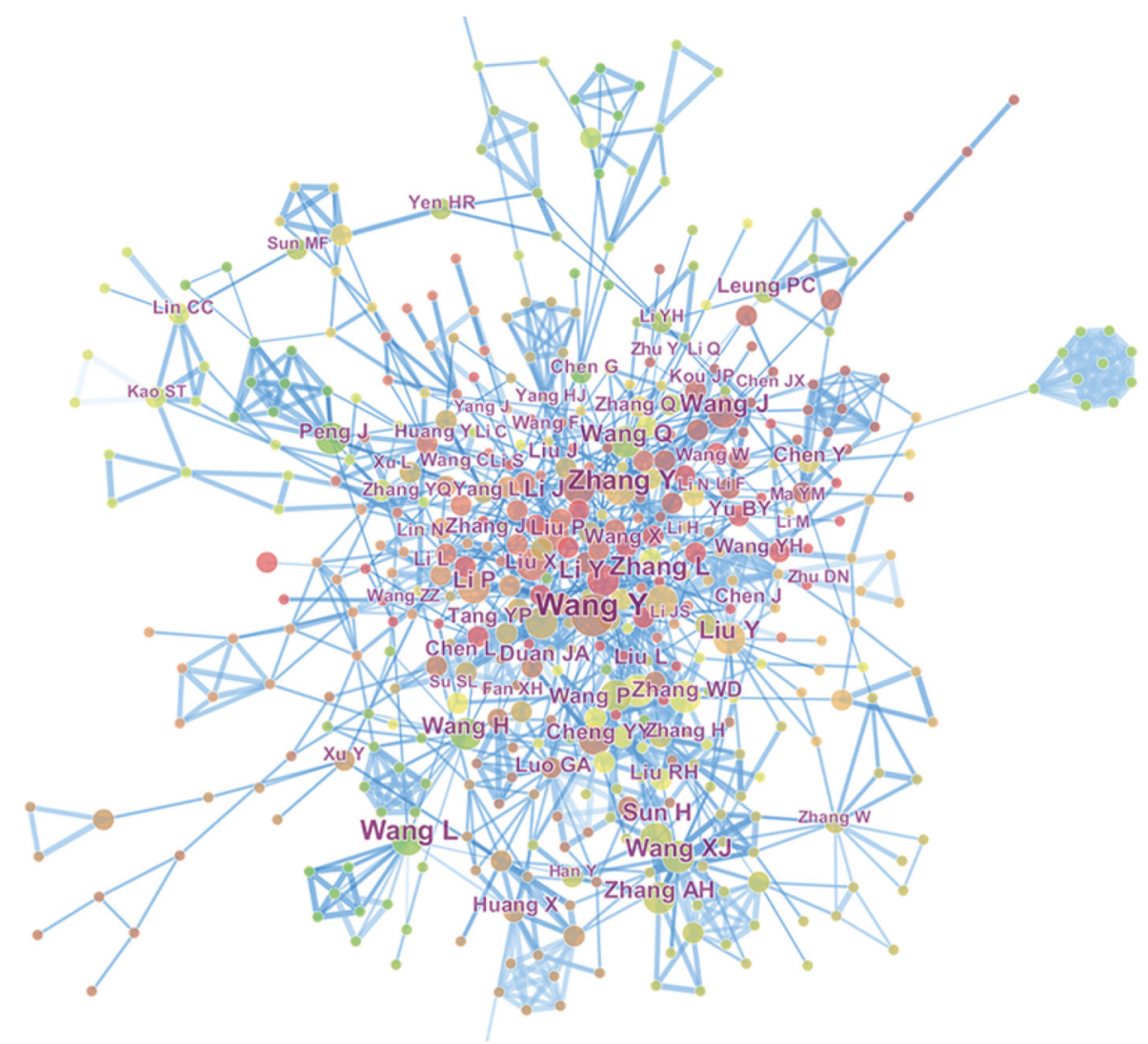

FIGURE 6: Coauthor map related to TCMF research from 2000 to 2016. A total of 536 nodes and 1621 connection lines were obtained. The size and position of nodes represent research frequency and the level of core of the author, respectively. The distance and connection of nodes represent the author's cooperation. The darkness of blue line represents the different years.

\section{Conclusion}

In summary, for future TCMF studies, we hope future scholars could diversify the research contents, extend the horizon of the cognitive perspectives of Traditional Chinese Medicine, and deepen the professional knowledge of TCMF research. For Traditional Chinese Medicine scholars, it is inappropriate to use the outcomes of treatment as the only standard to evaluate the effectiveness of TCMF. To make the breakthrough, the priority is to study the treatment mechanism of the disease on the selected formula and to clarify the effective components of the formula. This would enhance the authority and accuracy of TCMF study and help remove the stereotypes of Traditional Chinese Medicine as being "unscientific" and "empiricist". We also hope that with the improvement of the research level of TCMF, more best-quality papers will be published in important academic journals such as Nature and Science.

\section{Conflicts of Interest}

The authors declare that they have no conflicts of interest.

\section{Acknowledgments}

This work was supported by the National Natural Science Foundation of China (grant numbers 81741119, 81473542).
The authors are thankful to Tianjin University of Traditional Chinese Medicine for help in conducting this study.

\section{Supplementary Materials}

Japanese and South Korean research on Traditional Chinese Medicine Formula were compared with Chinese research, listing more than 60 kinds of Traditional Chinese Medicine Formulae in Roman alphabet match table according to Chinese Pinyin, Japanese Romaji, and South Korean Romaji to facilitate the exchange among researchers in the above three countries. (Supplementary Materials)

\section{References}

[1] World Health Organization (WHO), "WHO traditional medicine strategy: 20142023,” 2013, http://www.portal.pmnch .org/global_health_histories/seminars/Dr_Zhangs_Preentation_ GHHSeminar_86.pdf.

[2] X. Zhai, Q. Wang, and M. Li, “Tu Youyou's Nobel Prize and the academic evaluation system in China," The Lancet, vol. 387, no. 10029, p. 1722, 2016.

[3] W. Jia, W.-Y. Gao, Y.-Q. Yan et al., “The rediscovery of ancient Chinese herbal formulas," Phytotherapy Research, vol. 18, no. 8, pp. 681-686, 2004. 
[4] C. Hsiao, H. Tsou, Y. Wu, C. Lin, and Y. Chang, "Translation in different diagnostic procedures-traditional chinese medicine and western medicine," Journal of the Formosan Medical Association, vol. 107, no. 12, pp. S74-S85, 2008.

[5] OECD, “The OECD glossary of statistical terms," 2008, http:// stats.oecd.org/glossary/index.htm.

[6] N. A. Kazakis, "Bibliometric evaluation of the research performance of the Greek civil engineering departments in National and European context," Scientometrics, vol. 101, no. 1, pp. 505$525,2014$.

[7] A. Schubert and T. Braun, "Relative indicators and relational charts for comparative assessment of publication output and citation impact," Scientometrics, vol. 9, no. 5-6, pp. 281-291, 1986.

[8] C. Chen, "CiteSpace II: Detecting and visualizing emerging trends and transient patterns in scientific literature," Journal of the Association for Information Science and Technology, vol. 57, no. 3, pp. 359-377, 2006.

[9] J. I. De Granda-Orive, A. Alonso-Arroyo, and F. Roig-Vázquez, "Which data base should we use for our literature analysis? Web of Science versus SCOPUS," Archivos de Bronconeumología, vol. 47, no. 4, p. 213, 2011.

[10] C. Chen and J. C. Li, Text Mining and Visualization in Scientific Literature, Capital University of Economisc Business Press, Beijing, China, 2016.

[11] M. Xi, C. Hai, H. Tang, M. Chen, K. Fang, and X. Liang, "Antioxidant and antiglycation properties of total saponins extracted from traditional Chinese medicine used to treat diabetes mellitus," Phytotherapy Research, vol. 22, no. 2, pp. 228237, 2008.

[12] L. Castro-Vazquez, M. E. Alañón, V. Rodríguez-Robledo et al., "Bioactive flavonoids, antioxidant behaviour, and cytoprotective effects of dried grapefruit peels (Citrus paradisi macf.)," Oxidative Medicine and Cellular Longevity, vol. 2016, Article ID 8915729, 12 pages, 2016.

[13] A. E. Abd El-Wahab, D. A. Ghareeb, E. E. M. Sarhan, M. M. Abu-Serie, and M. A. El Demellawy, "In vitro biological assessment of berberis vulgaris and its active constituent, berberine: antioxidants, anti-acetylcholinesterase, anti-diabetic and anticancer effects," BMC Complementary and Alternative Medicine, vol. 13, no. 1, pp. 1-12, 2013.

[14] P. Farris, M. Yatskayer, N. Chen, Y. Krol, and C. Oresajo, "Evaluation of efficacy and tolerance of a nighttime topical antioxidant containing resveratrol, baicalin, and vitamin $\mathrm{E}$ for treatment of mild to moderately photodamaged skin," Journal of Drugs in Dermatology, vol. 13, no. 12, pp. 1467-1472, 2014.

[15] H. P. F. Peters and A. F. J. Van Raan, "Structuring scientific activities by co-author analysis - An expercise on a university faculty level," Scientometrics, vol. 20, no. 1, pp. 235-255, 1991.

[16] S. H. Zyoud, S. W. Al-Jabi, and W. M. Sweileh, "Scientific publications from arab world in leading journals of integrative and complementary medicine: a bibliometric analysis," BMC Complementary and Alternative Medicine, vol. 15, no. 1, p. 308, 2015.

[17] F. Danhier, O. Feron, and V. Préat, "To exploit the tumor microenvironment: passive and active tumor targeting of nanocarriers for anti-cancer drug delivery," Journal of Controlled Release, vol. 148, no. 2, pp. 135-146, 2010.

[18] V. Fuster, B. B. Kelly, and R. Vedanthan, "Promoting global cardiovascular health: moving forward," Circulation, vol. 123, no. 15, pp. 1671-1678, 2011.

[19] M. Zhou, H. Wang, and J. Zhu, "Cause-specific mortality for 240 causes in China during 1990-2013: a systematic subnational analysis for the global burden of disease study 2013," Lancet, vol. 387, no. 10015, pp. 251-272, 2016.

[20] R. J. King and P. J. Grant, "Diabetes and cardiovascular disease: pathophysiology of a life-threatening epidemic," Herz, vol. 41, no. 3, pp. 184-192, 2016.

[21] D. Mitanchez, A. Burguet, and U. Simeoni, "Infants born to mothers with gestational diabetes mellitus: Mild neonatal effects, a long-term threat to global health," Journal of Pediatrics, vol. 164, no. 3, pp. 445-450, 2014.

[22] S. Ross, A. Bossis, J. Guss et al., "Rapid and sustained symptom reduction following psilocybin treatment for anxiety and depression in patients with life-threatening cancer: A randomized controlled trial," Journal of Psychopharmacology, vol. 30, no. 12, pp. 1165-1180, 2016.

[23] T. D. Wachs, M. M. Black, and P. L. Engle, "Maternal depression: a global threat to children's health, development, and behavior and to human rights," Child Development Perspectives, vol. 3, no. 1, pp. 51-59, 2009.

[24] A. Mandegary, M. Pournamdari, F. Sharififar, S. Pournourmohammadi, R. Fardiar, and S. Shooli, "Alkaloid and flavonoid rich fractions of fenugreek seeds (Trigonella foenum-graecum L.) with antinociceptive and anti-inflammatory effects," Food and Chemical Toxicology, vol. 50, no. 7, pp. 2503-2507, 2012.

[25] C. Mo, L. Wang, J. Zhang et al., "The crosstalk between Nrf2 and AMPK signal pathways is important for the anti-inflammatory effect of Berberine in LPS-stimulated macrophages and endotoxin-shocked mice," Antioxidants \& Redox Signaling, vol. 20, no. 4, pp. 574-588, 2014.

[26] J. Lu, J.-S. Wang, and L.-Y. Kong, "Anti-inflammatory effects of Huang-Lian-Jie-Du decoction, its two fractions and four typical compounds," Journal of Ethnopharmacology, vol. 134, no. 3, pp. 911-918, 2011.

[27] A. R. Aroor, S. McKarns, V. G. Demarco, G. Jia, and J. R. Sowers, "Maladaptive immune and inflammatory pathways lead to cardiovascular insulin resistance," Metabolism - Clinical and Experimental, vol. 62, no. 11, pp. 1543-1552, 2013.

[28] H. Jaeschke, "Reactive oxygen and mechanisms of inflammatory liver injury: present concepts," Journal of Gastroenterology and Hepatology, vol. 26, supplement 1, pp. 173-179, 2011.

[29] S. P. Leighton, L. Nerurkar, R. Krishnadas, C. Johnman, G. J. Graham, and J. Cavanagh, "Chemokines in depression in health and in inflammatory illness: A systematic review and metaAnalysis," Molecular Psychiatry, vol. 23, no. 1, pp. 48-58, 2018.

[30] P. Guo, J. Wang, and H. Wang, "Study of the effects of Bu Yang Huan Wu Tang on SOD and MDA brain tissue in cerebral ischemia and reperfusion of rats," Chinese Journal of Applied Physiology, vol. 18, no. 2, pp. 108-131, 2002.

[31] J. Wang, K. Yao, X. Yang et al., "Chinese patent medicine Liu Wei Di Huang Wan combined with antihypertensive drugs, a new integrative medicine therapy, for the treatment of essential hypertension: A systematic review of randomized controlled trials," Evidence-Based Complementary and Alternative Medicine, vol. 2012, Article ID 714805, 7 pages, 2012.

[32] J.-H. Chiu, C.-J. Chang, J.-C. Wu et al., "Screening to identify commonly used chinese herbs that affect ERBB2 and ESR1 gene expression using the human breast cancer MCF-7 cell line," Evidence-Based Complementary and Alternative Medicine, vol. 2014, Article ID 965486, 11 pages, 2014.

[33] F. Li, X. Fan, Y. Zhang et al., "Cardioprotection by combination of three compounds from ShengMai preparations in mice with myocardial ischemia/reperfusion injury through AMPK 
activation-mediated mitochondrial fission," Scientific Reports, vol. 6, no. 1, Article ID 37114, 2016.

[34] X. Yang, X. Xiong, G. Yang, and J. Wang, "Chinese patent medicine Xuefu Zhuyu capsule for the treatment of unstable angina pectoris: a systematic review of randomized controlled trials," Complementary Therapies in Medicine, vol. 22, no. 2, pp. 391-399, 2014.

[35] L. Yan, Q. Hu, M. S. Mak et al., "A Chinese herbal decoction, reformulated from Kai-Xin-San, relieves the depression-like symptoms in stressed rats and induces neurogenesis in cultured neurons," Scientific Reports, vol. 6, no. 1, Article ID 30014, 2016.

[36] Y. Zhang, M. Han, Z. Liu, J. Wang, Q. He, and J. Liu, "Chinese herbal formula Xiao Yao San for treatment of depression: a systematic review of randomized controlled trials," EvidenceBased Complementary and Alternative Medicine, vol. 2012, Article ID 931636, 13 pages, 2012.

[37] I.-M. Liu, T.-F. Tzeng, and S.-S. Liou, "A Chinese herbal decoction, Dang Gui Bu Xue Tang, prepared from Radix Astragali and Radix Angelicae sinensis, ameliorates insulin resistance induced by A high-fructose diet in rats," EvidenceBased Complementary and Alternative Medicine, vol. 2011, Article ID 248231, 11 pages, 2011.

[38] Y.-L. Yu, S.-S. Lu, S. Yu et al., "Huang-Lian-Jie-Du-Decoction modulates glucagon-like peptide-1 secretion in diabetic rats," Journal of Ethnopharmacology, vol. 124, no. 3, pp. 444-449, 2009.

[39] C. Kelleher and T. Wagener, "Ten guidelines for effective data visualization in scientific publications," Environmental Modeling and Software, vol. 26, no. 6, pp. 822-827, 2011.

[40] C. Chen, "Science mapping: a systematic review of the literature," Journal of Data and Information Science, vol. 2, no. 2, pp. 1-40, 2017. 


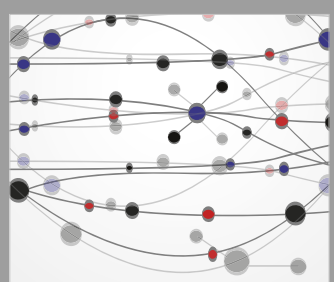

The Scientific World Journal
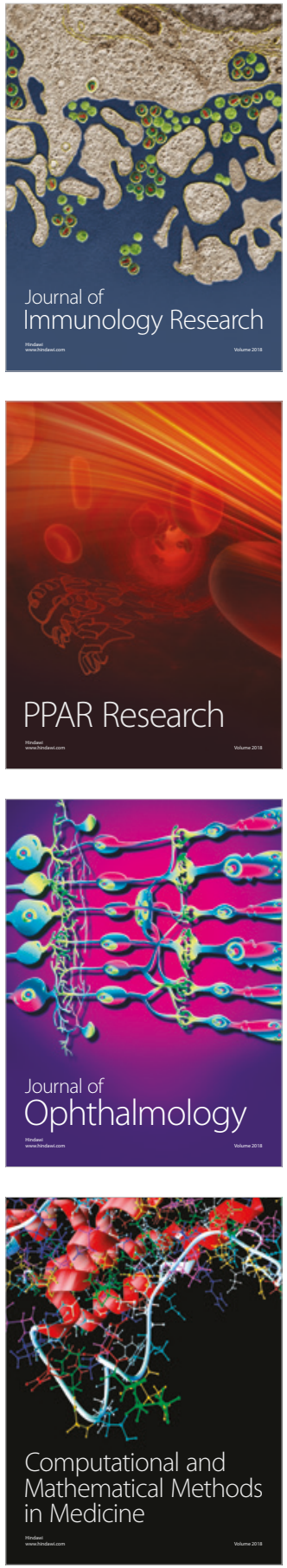

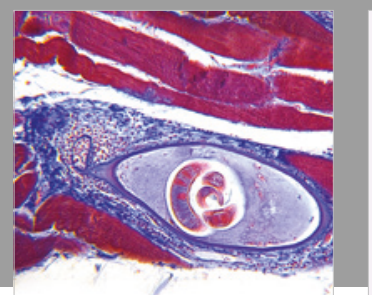

Gastroenterology Research and Practice

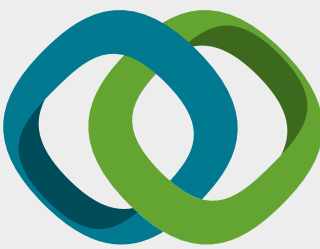

\section{Hindawi}

Submit your manuscripts at

www.hindawi.com
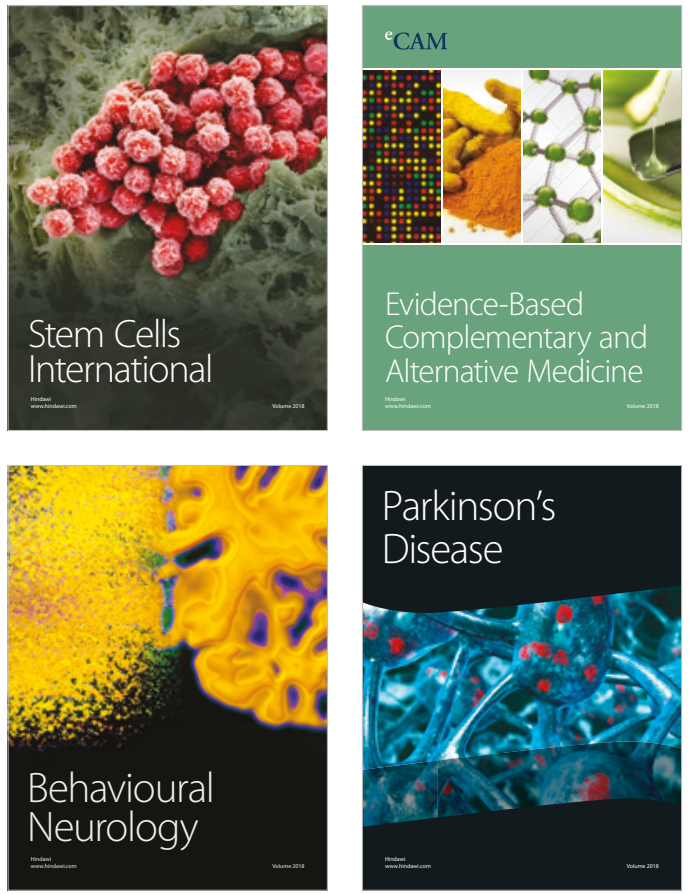

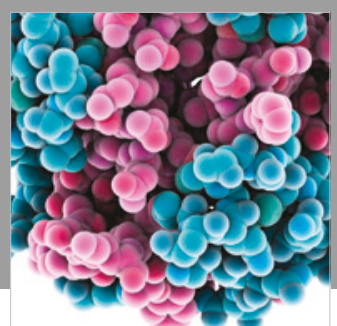

ournal of

Diabetes Research

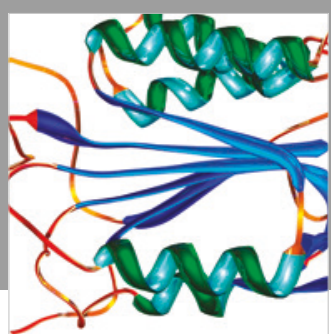

Disease Markers
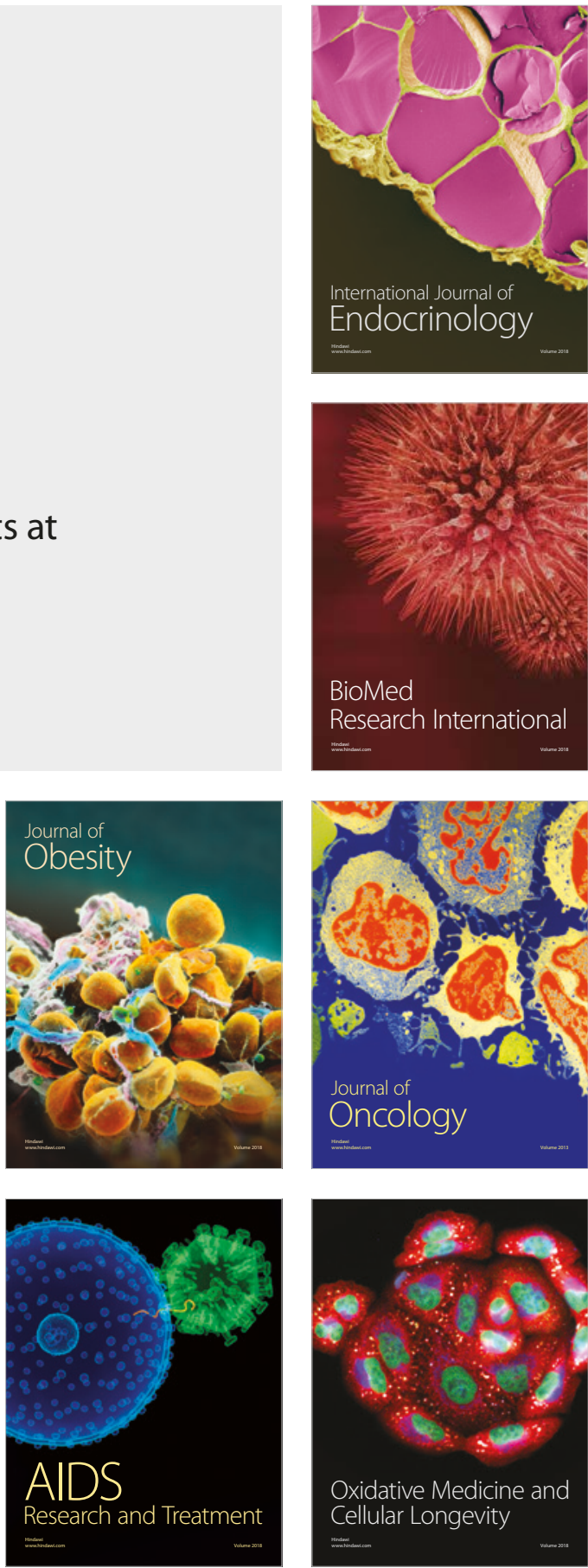